\title{
Unmet needs for community-based physiotherapy services for adults in Canada and the United States: A scoping review protocol
}

\author{
Alison Hock, Justis Beley, Michaela Bennett, Nick Buitelaar, Amy Connell, Kate Rickaby, Liliana Coman and Sarah Wojkowski* \\ Physiotherapy Program, School of Rehabilitation Science, McMaster University, Hamilton ON, Canada
}

\begin{abstract}
Introduction: Inadequate access to healthcare resources resulting in unmet needs is a global issue that needs to be addressed. Canada and the United States have substantially distinct healthcare systems that have the potential to differ in the prevalence and presentation of "unmet needs." Physiotherapists, in collaboration with other healthcare professionals, are in a unique position to contribute to closing gaps in access.

Purpose: The purpose of this scoping review is to explore unmet needs for community-based physiotherapy services in adults in Canada and the United States.

Methods: A scoping review methodology will be used to guide an in-depth literature search in three electronic databases: Ovid MEDLINE, Ovid EMBASE and EBSCOhost CINAHL. All titles and abstracts identified by the search will be screened by two independent reviewers, according to specific inclusion criteria such as language, publication date and population. The full texts of articles which are considered to meet all inclusion criteria will be reviewed in Covidence, by two independent reviewers and assessed for inclusion in the scoping review. Data will then be extracted from each article by two independent reviewers, using a data extraction table. This extracted data will be displayed in tables and results will be connected to the objective of this review through a narrative summary.
\end{abstract}

Conclusions: The data collected from the proposed review will explore unmet needs for adult physiotherapy services in the community. Potential barriers to access, as well as shared and unique unmet needs in Canada and the United States will be identified.

\section{Introduction}

Healthcare is available through various systems and access points. However, differences in economic status can create disparities in access to healthcare services [1]. It is not the responsibility of a sole proprietor, but rather all countries, to raise more funds, or diversify funding sources, to relieve the burden of unmet needs in healthcare [2]. However, it is the government's duty to ensure that all public and private healthcare providers operate efficiently to fulfil patient needs [2]. Schneider et al. compared 11 high-income countries on 72 indicators of performance in five domains of healthcare: process, access, administrative efficiency, equity, and outcomes [3]. The United States (US) was ranked last, receiving particularly poor scores in access and affordability. Canada's results were also substandard, ranking ninth overall, with its worst score in the access domain [3]. Furthermore, the US and Canada were identified as having large disparities in access between low- and high-incomes, particularly due to financial barriers [3]. In this context, patients may choose to forgo treatments or tests, due to associated costs [3].

While geographically close, Canada and the US have unique healthcare systems. Canada has a single-payer, publicly funded healthcare system, while the US is primarily a privatized multipayer system [4]. Healthcare services are costly and consume a large proportion of budgets across all countries [5]. According to a 2013 study by the Organization for Economic Co-operation and Development (OECD), the US spent more than twice as much on health expenditures compared to Canada [6]. Despite this large financial investment, a crisis for access and equity remains in the US [7]. Recent estimates suggest that nearly $14 \%$ of US residents are uninsured, with rates being higher among people of low socio-economic status [7]. According to a 2017 US national report, approximately $40 \%$ of adults reported lacking a usual source of care, with nearly $15 \%$ of those individuals identifying financial barriers as a primary reason [7]. In Canada, the impact of chronic disease continues to shape the healthcare system, highlighting the importance of accessible healthcare resources in this population [5]. Wojkowski et al. completed an analysis of unmet physiotherapy needs in Canadian communities and found that those with chronic diseases had inconsistent access to, and delivery of services [8]. Those who reported access difficulties cited cost and lack of availability as reasons for these challenges [8]. A recent Canadian pilot project explored patient perspectives of the barriers to accessing physical therapy services and the resulting unmet needs in a population experiencing poverty and homelessness [9]. The results of this project indicated barriers to access in this population included participants' lack of knowledge on their condition, cost, transportation, and wait time [9].

${ }^{*}$ Correspondence to: Sarah Wojkowski, Physiotherapy Program, School of Rehabilitation Science, McMaster University, Hamilton ON, Canada, E-mail: wojkows@mcmaster.ca

Key words: Canada, United States, physiotherapy, physical therapy, community, unmet needs

Received: March 10, 2020; Accepted: March 17, 2020; Published: March 26 , 2020 
The World Health Organization (WHO) defines universal health coverage as the ability to access various levels of health services, including health promotion, prevention, treatment, rehabilitation and palliation [10]. Physiotherapists have a unique role in the healthcare system by way of providing intervention at each of these levels [11] to optimize functional abilities and quality of life across musculoskeletal, neurological, and cardiovascular systems $[12,13]$. Physiotherapists are ideal members of primary healthcare teams because they are educated on chronic health challenges and their subsequent impact on patient function, and thus are in a position to enhance healthcare access in atrisk populations. Stafford and Wood (2017) proposed that improving health disparities should be viewed from both health and social perspectives by incorporating the social determinants of health [14], and physiotherapists are well positioned to understand both of these perspectives.

The purpose of this scoping review is to explore the unmet needs for community-based physiotherapy services for adults in Canada and the US. There is no universally accepted definition of unmet need and it can be identified from varying perspectives, including those of the patient and healthcare provider [15]. For the purposes of this paper, we have chosen to use Bradshaw's taxonomy of social need and McIntyre's framework of levels of access to health services $[16,17]$ to define need and unmet need, respectively. Bradshaw (1972) describes "need" with four interrelated levels: normative need is the need defined by an expert or professional; felt need is equated to want; expressed need is described as felt need turned into action; and comparative need, which compares the characteristics of the population who receives a service [16]. McIntyre et al. propose a framework that outlines three levels of access to health services: availability (of the services or providers), affordability (costs and impact of those costs), and acceptability (attitudes, beliefs, and expectations between patient and provider) [17]. Using Bradshaw's levels of "need" as a foundation, McIntyre's access framework will be used to identify how and why physiotherapy needs are "unmet" for adults in Canada and the US $[16,17]$. Based on our findings, we will explore the ways by which needs for physiotherapy services are unmet and identify potential barriers to access in both Canada and the US. Data from Canada and the US will also be compared and contrasted to provide insight into shared and unique areas for improvement in both countries.

\section{Review Question}

This scoping review will address the following question: "What are the unmet needs for community-based physiotherapy services for adults in Canada and the United States?"

\section{Inclusion Criteria}

Participants: This scoping review will include articles that address unmet needs for adult populations (defined as 18 years and older).

Concept: The concepts studied in this review are the unmet needs or perceived needs for community-based physiotherapy services for adults. This review will examine the gaps in physiotherapy services administered to adults in a community setting.

Context: This review will consider studies that evaluate the unmet needs for services, interventions, treatments or management delivered by a physiotherapist, in a community-based setting (defined as being administered to individuals living independently, including outpatient and home care services). Studies conducted in Canada and the United States will be included in this review.

\section{Types of Sources}

As per the scoping review protocol by Hanna et al. both quantitative and qualitative study designs will be considered for inclusion in this scoping review [13]. This will include experimental and quasiexperimental designs such as randomized and non-randomized control trials, single-subject studies, and pre-test post-test studies [13]. Examples of qualitative study designs that will be considered include interpretive description, narrative inquiry, phenomenology, ethnography, grounded theory, and action research studies [13]. Observational studies will also be considered, including, but not limited to, prospective cohort studies, retrospective cohort studies, cross-sectional studies, case-control studies, and case-series [13]. This scoping review will also consider systematic reviews, meta-analyses, and scoping reviews [13].

Proposed Eligibility Criteria: This review will include articles that were published within the last five years (2015 to present). The choice to exclude articles published prior to 2015 will allow our results and discussion to reflect the most recent changes in healthcare funding models as well as the current political climate within Canada and the United States $[18,19]$. Articles written in English, French, and Spanish will be considered.

\section{Methods}

The reporting of this scoping review protocol (Appendix 1) as well as the final scoping review will be developed in accordance with the PRISMA Extension for Scoping Reviews (PRISMA-ScR) checklist (Appendix 2). The following stages, proposed by Arksey and O'Malley, will guide our scoping review [20].

1. Research question development, incorporating population, concept and context framework.

2. Scoping review protocol publication.

3. Search strategy generation, literature search, and identification of studies for screening.

4. Study selection, using inclusion criteria.

5. Detailed analysis of identified studies, data extraction and charting.

6. Summarization, collation, reporting, and presentation of results.

\section{Search Strategy}

A preliminary search was conducted in Ovid EMBASE (1996-February 2020), in which the key terms of physiotherapy, physical therapy and unmet needs were used. To fully capture the concept of unmet rehabilitation service needs, additional terms were used, including health service's needs, perceived needs, and access to care. As the focus of this scoping review is on services offered in a community setting, the terms used to capture this concept included outpatient, community health services, home care services, and independent living. To address the comparison between Canada and the US, the terms North America, Canada, and the United States, as well as each state, province, and territory within each country were included. The search was limited to English, French, and Spanish languages, and studies published between 2015 and the present. Finally, to ensure all concepts were thoroughly captured, a McMaster Health Sciences librarian was consulted, and additional search terms were included to further develop the search strategy. A full search strategy for the preliminary search of Ovid EMBASE is provided in (Appendix 3). 
Information Sources: A comprehensive search of the literature will be conducted in three online databases. These will include Ovid MEDLINE (1996-February 2020), Ovid EMBASE (1996-February 2020), and EBSCOhost CINAHL (1937-February 2020).

\section{Study Selection}

Upon generating the complete search strategy in all three databases, each of the identified articles will be uploaded into Covidence, a review management software, where duplicates will be removed. All titles and abstracts will be screened by two independent reviewers, according to previously outlined inclusion criteria. Titles or abstracts that do not explicitly address physiotherapy or services delivered to adults will be excluded. The full texts of articles which are considered to meet all inclusion criteria will be reviewed by two independent reviewers in Covidence and assessed for inclusion in the scoping review. Articles that do not meet full criteria will be excluded, with reasons for exclusion clearly provided in an appendix of the final scoping review. A kappa (K) statistic, a measure of inter-rater reliability and agreement between the two reviewers, will be calculated $[13,21]$. Any disagreements that arise will be resolved through discussion between reviewers or the involvement of a third reviewer [13]. The final scoping review will include a full report of search results, listed in a PRISMA flow diagram [13].

\section{Data Extraction}

Data will be extracted from each article that has been selected for inclusion in this scoping review by two independent reviewers, using the data extraction table developed for this purpose. Extracted data will include study details regarding population, design, methods, description of unmet needs, and important findings related to each component of the population, concept, context framework. A draft version of this extraction table is provided and will be adapted and altered as needed, during the data extraction process. Any disagreements that may arise at the stage of data extraction will be resolved through discussion between reviewers, or the involvement of a third reviewer. Finally, pairs of reviewers will review each article's risk of bias and come to a consensus through discussion, in cases where there is disagreement. An estimate of agreement between reviewers, beyond chance, will be calculated and presented using kappa.

\section{Data Presentation:}

Extracted data that is relevant to the scoping review research question will be clearly presented in tables. The results will also be connected back to the objective of this review through a narrative summary.

\section{Conclusion}

This scoping review protocol outlines how we plan to search the current literature to discover trends in unmet needs for adult physiotherapy services in the community within Canada and the US. We also plan to compare and contrast access to physiotherapy services in Canada and the US, in order to provide some insight into areas for improvement in both countries. Upon completion of our review, we plan on publishing the results, presenting the information to other healthcare professionals, and using it to inform our own practices. We hope that through sharing our results, healthcare providers will have an improved understanding of the current unmet needs so that we all may play a part in minimizing, and eventually eliminating them. We also hope that this insight will improve the quality of advocacy for the physiotherapy profession when addressing unmet needs.

\section{Funding}

This research did not receive any grants from funding agencies in the public, commercial, or not-for-profit sectors. Non-financial sources of support are provided by the scoping review's sponsor, McMaster University. The sponsor is not involved in the conduction of the scoping review. McMaster University will provide study authors who have access to various resources which include obtaining access to databases and articles as needed.

Declaration of Conflicting Interests: The authors have no conflicts of interest to disclose.

Acknowledgements: We would like to acknowledge Ms. Neera Bhatnagar, Health Sciences Librarian at McMaster Health Sciences Library for assisting in the development of the search strategy by sharing her knowledge and expertise. The authors declare that the individuals who received acknowledgments did not receive funding for their assistance and did not provide assistance with the writing process.

Contributions: All authors associated contributed to the development of the research question and selection criteria. SW and LC provided insight into the methodology of developing a scoping review. $\mathrm{NB}$ and $\mathrm{AC}$ developed the search strategy and conducted the search. AC, AH, JB, MB and KR drafted the manuscript. All authors contributed to providing feedback and approving the final manuscript.

\section{References}

1. Dickman SL, Himmelstein DU, Woolhandler S (2017) Inequality and the health-care system in the USA. Lancet 389: 1431-1441. [Crossref]

2. World Health Organization (2010) The world health report. Health systems financing the path to universal coverage. Geneva: WHO Press.

3. Schneider E, Sarnak D, Squires D, Shah A, Doty M (2017) Mirror, Mirror 2017 international comparison reflects flaws and opportunities for better health care. U.S National Library of Medicine.

4. O'Neill J, O'Neill D (2007) Health status, health care and inequality: Canada vs. the U.S. Forum for Health Economics \& Policy, Berkeley Electronic Press.

5. Martinello N, Bhandari A, Santos J, Dinh T (2017) The role of physiotherapy in Canada: contributing to a stronger health care system. Ottawa: The Conference Board of Canada.

6. DPE (2016) The U.S. Health Care System: An International Perspective. Department for professional employees.

7. Joynt Maddox KE, Bauchner H, Fontanarosa PB (2019) US health policy-2020 and beyond: introducing a new JAMA series. JAMA 321: 1670-1672. [Crossref]

8. Wojkowski S, Smith J, Richardson J, Birch S, Boyle M (2016) A scoping review of need and unmet need for community-based physiotherapy in Canada. J Crit Rev 3: $17-23$.

9. Oosman S, Weber G, Ogunson M, Bath B (2019) Enhancing access to physical therapy services for people experiencing poverty and homelessness: the lighthouse pilot project. Physiother Can 71: 176-186. [Crossref]

10. Evans DB, Hsu J, Boerma T (2013) Universal health coverage and universal access Bull World Health Organ 91: 546-546A. [Crossref]

11. World Confederation for Physical Therapy (2020) What is physical therapy?

12. Ontario Physiotherapy Association (2017) Physiotherapy in primary health care. Toronto: The Association.

13. Hanna S, Lyons M, Parker J, Stokes A (2019) Unmet needs for physiotherapy services for the pediatric population in Canada: a scoping review protocol. Phys Med Rehabil 4: $1-5$.

14. Stafford A, Wood L (2017) Tackling health disparities for people who are homeless? Start with social determinants. Int J Environ Res Public Health 14: 1535. [Crossref]

15. Smith S, Connolly S (2019) Re-thinking unmet need for health care: introducing a dynamic perspective. Health Econ Policy Law 25: 1-18. [Crossref] 
Hock A (2020) Unmet needs for community-based physiotherapy services for adults in Canada and the United States: A scoping review protocol

16. Bradshaw J (1972) Taxonomy of social need. In: McLachlan, Gordon. Problems and progress in medical care: essays on current research, 7th series. Oxford University Press-London.

17. McIntyre D, Thiede M, Birch S (2009) Access as a policy-relevant concept in low- and middle-income countries. Health Econ Policy Law 4: 179-193. [Crossref]

18. Vogenberg F (2019) US healthcare trends and contradictions in 2019. Am Health Drug Benefits 12: 40-47. [Crossref]
19. Tam P (2015) Canada 2020 health summit report.

20. Arksey H, O’Malley L (2007) Scoping studies: towards a methodological framework. Int J Soc Res Method 8: 19-32.

21. McHugh ML (2020) Interrater reliability: the kappa statistic. Biochem Med (Zagreb) 22: 276-282. [Crossref]

Copyright: (C2020 Hock A. This is an open-access article distributed under the terms of the Creative Commons Attribution License, which permits unrestricted use, distribution, and reproduction in any medium, provided the original author and source are credited. 